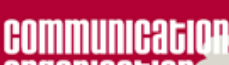
Oryanllatín.

\section{Communication et organisation}

Revue scientifique francophone en Communication organisationnelle

\section{$53 \mid 2018$}

Digitalisation et recrutement : perspectives

informationnelles et communicationnelles

\title{
Dawna BALLARD et Matthew MCGLONE (eds.), Work Pressures: New Agendas in Communication
}

New York : Routledge, 2016, 170 p.

Delphine Dupré

\section{QpenEdition}

\section{Journals}

Édition électronique

URL : https://journals.openedition.org/communicationorganisation/6488

DOI : 10.4000/communicationorganisation. 6488

ISSN : 1775-3546

Éditeur

Presses universitaires de Bordeaux

Édition imprimée

Date de publication : 1 juin 2018

Pagination : 243-244

ISBN : 979-10-300-0302-4

ISSN : 1168-5549

Référence électronique

Delphine Dupré, « Dawna BALLARD et Matthew MCGLONE (eds.), Work Pressures : New Agendas in

Communication », Communication et organisation [En ligne], 53 | 2018, mis en ligne le 01 juin 2018, consulté le 29 janvier 2022. URL : http://journals.openedition.org/communicationorganisation/6488 ; DOI : https://doi.org/10.4000/communicationorganisation.6488 
La cinquième et dernière partie du livre porte sur les théories féministes. Dans une optique épistémologique, les principales contributions des féministes aux études en communication médiatique sont développées, tout en dressant un portrait de leur histoire. Le lecteur pourra prendre connaissance du contexte sociohistorique et intellectuel de ce mouvement et de quelques figures marquantes des vagues successives du féminisme en France et dans les pays anglo-saxons. Un dernier chapitre sur le féminisme poststructuraliste expose les tensions des approches critiques sur le genre et la communication.

Le caractère distinctif, et réussi, de cet ouvrage est de proposer une synthèse des avancées théoriques et scientifiques des approches critiques en communication, sans s'écarter du souci constant de valoriser les éléments potentiels pour une recherche en communication.

Claire D'HENNEZEL ${ }^{5}$

\section{Dawna BALLARD et Matthew MCGLONE (eds.), Work Pressures: New Agendas in Communication. New York : Routledge, 2016, $170 \mathrm{p}$.}

Work pressures: new agendas in communication est un ouvrage collectif structuré en 3 parties. Les premiers chapitres portent sur le concept de surcharge informationnelle. Dans la deuxième partie de l'ouvrage, les auteurs étudient l'impact du stress et des accélérations temporelles sur la communication organisationnelle. Enfin, les derniers chapitres explorent les différentes manières dont ces bouleversements organisationnels s'actualisent dans la communication médiatisée par ordinateur (CMC).

Le postulat de base de cet ouvrage est qu'une nouvelle conception du temps a émergé au $\mathrm{XXI}^{\mathrm{e}}$ siècle. La grande horloge, allégorie de l'organisation du travail du $20^{\mathrm{e}}$, représente aujourd'hui un symbole désuet. Au temps linéaire et clairement balisé du taylorisme se sont substituées de nouvelles temporalités professionnelles. Ces dernières ont contribué à la propagation du syndrome de surcharge informationnelle, concept qui

5- Claire D'Hennezel est docteure en sciences de l'information et de la communication et chercheure associée au laboratoire MICA (EA 4426) de l'Université Bordeaux Montaigne. Ses travaux portent sur l'intelligence économique, la veille, la communication des organisations, l'ubérisation et l'innovation organisationnelle. Elle enseigne par ailleurs depuis de nombreuses années comme professeure certifiée en Économie Gestion. 
désigne un déséquilibre entre la quantité d'informations à traiter et les ressources pour $\mathrm{y}$ parvenir.

Ce concept est défini de manière assez floue et approximative dans la littérature scientifique. Toutefois, sa récurrence dans le discours des acteurs en fait un sujet de recherche légitime, répondant à une urgence sociale. La surcharge informationnelle est préoccupante tant en raison de ses effets sur la santé des salariés que sur le fonctionnement de l'organisation. En effet, ce trouble dégraderait la capacité à se concentrer, à identifier les priorités et à prendre des décisions éclairées.

Les auteurs soulignent la tendance à blâmer uniquement les objets techniques. Or, la surcharge informationnelle est un phénomène complexe qui résulte de facteurs cognitifs, organisationnels, sociétaux et culturels. Plus particulièrement, l'engagement des salariés dans plusieurs projets simultanés en constituerait la cause principale. La dimension genrée de ce phénomène est également mise en exergue. En raison du partage inégal des tâches qui règne encore aujourd'hui dans la majorité des foyers en Amérique du Nord, les femmes seraient davantage exposées que les hommes.

Cette dimension genrée est également traitée dans un chapitre consacré au phénomène du burn out chez les médecins. Ce concept désigne un état mental et émotionnel provoqué par un stress éprouvé sur une longue période. Le burn out résulte de plusieurs facteurs individuels et organisationnels. En raison des attentes en termes de care, les femmes médecins consacreraient davantage de temps aux patients et seraient plus touchées par le burn out que leurs homologues masculins. L'auteure de ce chapitre précise que les régulations faisant intervenir la communication - participation des individus au processus de décision, amélioration du soutien social - se révèlent particulièrement efficaces. Or, les méthodes de prévention utilisées habituellement se concentrent principalement sur les causes individuelles du burn out.

Le thème des affects numériques est traité dans le dernier chapitre de l'ouvrage. Loin de constituer un média froid et neutre, Internet fournit aux interlocuteurs des outils pour exprimer leurs émotions. Les expérimentations menées par les auteurs révèlent que les émotions négatives, suscitées par le stress et la pression temporelle, transparaissent dans les échanges numériques. 
Pour conclure, work pressures est un ouvrage stimulant qui ouvre de nombreuses perspectives et pose plusieurs jalons dans la structuration d'une approche communicationnelle des temporalités professionnelles et de leurs effets délétères.

Delphine DUPRÉ ${ }^{6}$

6- Delphine Dupré est doctorante contractuelle au sein du laboratoire MICA (EA 4426) de l'Université Bordeaux Montaigne. Sa thèse porte sur les incivilités par messagerie électronique et s'inscrit dans le cadre du projet régional Civilinum coordonné par Valérie Carayol et Aurélie Laborde. 